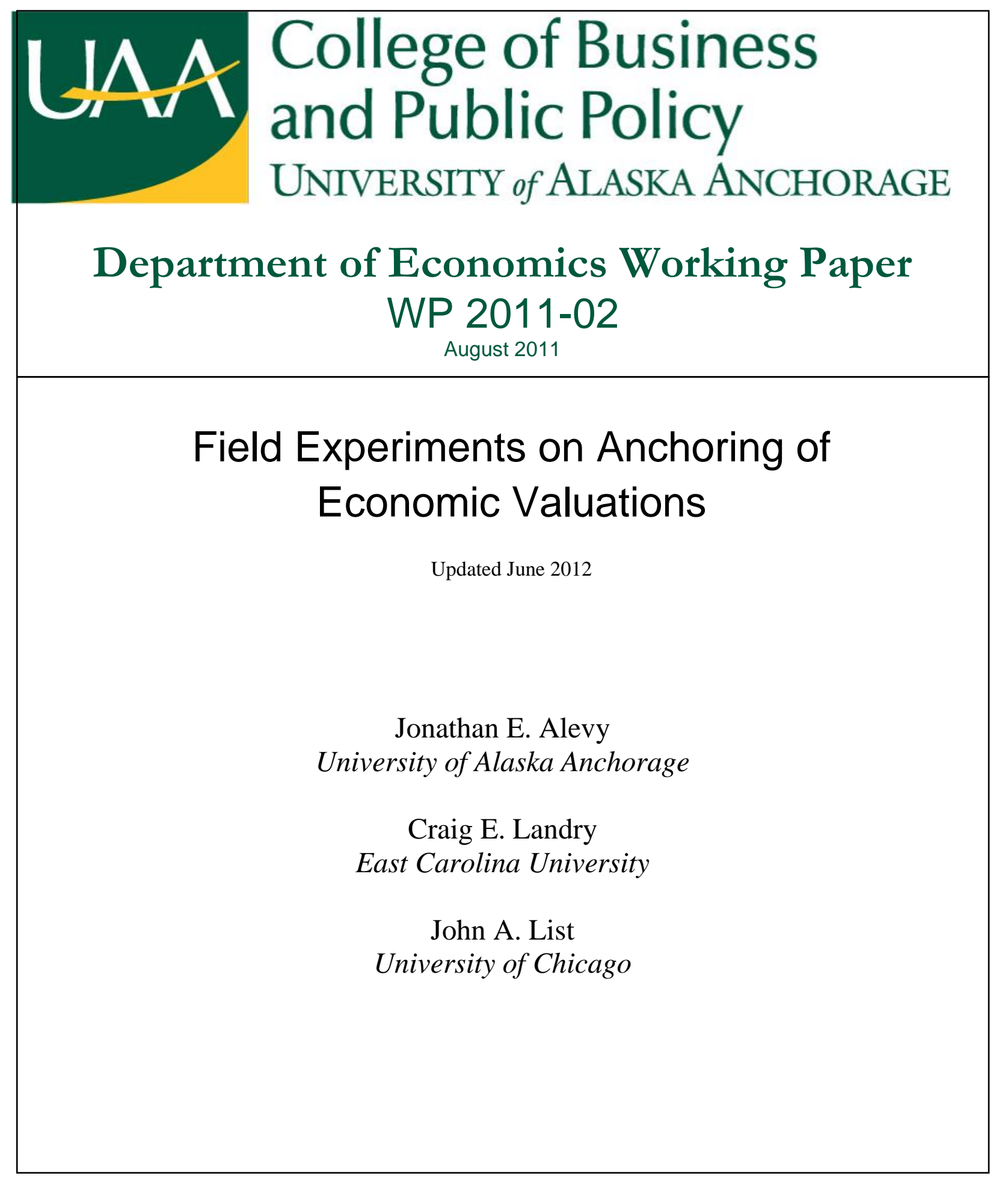

UAA DEPARTMENT OF ECONOMICS

3211 Providence Drive

Rasmuson Hall 302

Anchorage, AK 99508

http://www.cbpp.uaa.alaska.edu/econ/econhome.aspx 


\title{
Field Experiments on the Anchoring of Economic Valuations
}

\author{
Jonathan E. Alevy, Craig E. Landry, and John A. List* \\ June 2012
}

JEL: C93 (Field Experiments), D11 (Microeconomic Theory)

Key words: anchoring, valuation, field experiment, experience

\begin{abstract}
Alevy, Department of Economics, University of Alaska Anchorage; Landry, Department of Economics, East Carolina University; List, Department of Economics, University of Chicago, and Research Fellow, National Bureau of Economics Research
\end{abstract}




\title{
Field Experiments on Anchoring of Economic Valuations
}

\begin{abstract}
A pillar of behavioral research is that preferences are constructed during the process of choice. A prominent finding is that uninformative numerical "anchors" influence judgment and valuation. It remains unclear whether such processes influence market equilibria. We conduct two experiments that extend the study of anchoring to field settings. The first experiment produces evidence that inexperienced consumers can be anchored in the value elicitation process, yet there is little evidence that experienced agents are influenced by anchors. The second experiment finds that anchors have only transient effects on market outcomes which converge to equilibrium predictions after a few market periods.
\end{abstract}




\section{Field Experiments on Anchoring of Economic Valuations}

\section{Introduction}

Take the last three digits of your social security number. Turn those numbers into a dollar value (i.e., if your numbers are 462 then they provide a value of \$462). Consider whether you would be willing to pay that dollar amount for a first edition of JRR Tolkien's, The Hobbit. Now, how much would you actually pay for a first edition original copy? A stylized result from laboratory experiments in economics and psychology is that the answer to the latter valuation question is strongly influenced by the dollar amount computed at the start. ${ }^{\mathrm{i}}$

Critics of neoclassical theory have argued that the influence of uninformative anchors - such as the transformed social security number - refutes the notion that decision makers' preferences are pre-defined, consistent, and stable (e.g. Slovic 1995). A natural conclusion one might draw from studies of anchoring is that decisions may be influenced by irrelevant circumstances, and no optimization principles underlie even straightforward economic decisions.

Although the laboratory evidence consonant with anchoring effects is considerable, the importance of anchoring for economic outcomes remains an open question. ${ }^{\text {ii }}$ One reason is that many of the experiments lack meaningful incentives, and we believe economists are justifiably inclined to discount results that may merely reflect mistakes made by unmotivated subjects. Consistent with this view is the idea that, with appropriate incentives and feedback, anchoring effects should diminish and behavior more closely match predictions from neoclassical models. ${ }^{\text {iii }}$ Furthermore, even in those cases where inexperienced consumers constitute a considerable portion of the market, little is known about how such consumers influence equilibrium price and quantity.

This study provides some insight into these questions by conducting two complementary field experiments within a well-functioning marketplace: the sportscard market. In contrast to anchoring studies conducted in classroom or laboratory settings, the sportscard marketplace has a number of advantages. First, it is a natural setting for an examination of preference structures since it provides a rich array of subjects making decisions in a familiar environment. Second, we can identify factors that arise endogenously, such as market experience or a person's role in the marketplace, and 
impose the remaining controls necessary to implement a clean experiment to explore whether these or other factors foster or attenuate anchoring. While we do not consider the sportscard market particularly worthy of study in its own right, when larger or "more important" markets are not available for experiments with parallel control, manipulating smaller scale markets such as the sportscard market has value in that we can learn behavioral tendencies from at least one naturally-occurring setting. See Gneezy and Wu (2006) and Ariely et al. (2009) for further insights on these issues.

In our first field experiment, we make use of an anchoring protocol very similar to the one described in our opening paragraph. To give anchoring effects their best chance we exogenously vary the type of good, between subjects. The first good is a newly introduced variant into the market - an unopened package of sportscards. Given its recent introduction, no established market price for the good existed at the time of our experiment, but subjects entering the market did expect to buy, sell, and trade related goods. The second good - a jar of peanuts - is familiar, but unexpected in the sportscard marketplace, and ordinary consumers would not have expected to value such a good. The novelty is a crucial element in some recent behavioral models that surmise that choices will be more anomalous in situations that present themselves as surprises (see, e.g., the recent work of Koszegi and Rabin (2008)). By examining the valuations of both ordinary consumers and professional sports memorabilia dealers over both goods, we are able to explore whether market experience and market roles are associated with susceptibility to the anchoring protocol.

The data provide some unique insights. First, there is suggestive evidence that anchoring matters in the valuation exercise. For ordinary consumers the anchor influences valuations for the good that they did not expect to value when entering the market (the jar of peanuts), but they were not influenced by the anchor when valuing the good they expected to buy, sell, and trade in the market (the unopened package of sportscards). We find that dealers were not influenced by the anchor for either peanuts or the unopened package of sportscards. Pooling subjects across market roles to investigate market experience, we find that individuals with one year or less of market experience are influenced by the uninformative anchor. 
Our results suggest that a segment of the population in a naturally-occurring market could be susceptible to common anchoring effects. Accordingly, these findings should influence the extensive literature that has concentrated on finding non-expected utility resolutions to paradoxes of choice, for example. From a policy perspective, these results merit serious consideration in several circles. For instance, the effects of anchoring have been of interest to practitioners of cost-benefit analysis for decades. In the non-market valuation setting, evidence of anchoring effects has become an important heuristic for evaluating the reliability of stated preference methods such as contingent valuation that are used to estimate the value of public goods (e.g. Holmes and Kramer, 1995; Green et al., 1998; Bateman et al., 2008). Anchoring in this setting is consistent with the idea that the task of valuing non-market resources is hampered by consumers' unfamiliarity with such decision environments and the complexity of the exercise. Empirical results herein present some guidance into the underlying reasons for the observed anchoring.

While these behaviors should have import for survey based approaches to elicit non-market values, whether, and to what extent, these behavioral tendencies influence market equilibria is largely unknown. Extant theory suggests that factors such as the composition of marginal and inframarginal traders, the trading institution, and other market particulars, might have significance in the transference of the anchoring result to markets. To provide insight on this question, we design a second field experiment that makes use of a stronger anchoring treatment: in a decentralized bargaining market, we vary information concerning previous transaction prices, which serve as the focal source of market uncertainty. We find evidence that the anchor has some influence on early market transactions, but that the effect is transient. Even in those cases where the market is populated entirely by inexperienced consumers, quantities and prices approach the intersection of supply and demand after a few rounds of market play.

Taken together, the results in this paper suggest that when constant feedback mechanisms are in place and participants are able to receive signals of value and adjust their behavior accordingly, anchoring does not play a significant role in bilateral market outcomes. In other important instances where feedback is limited, such as contingent valuation exercises commonly performed by government agencies, anchoring can have 
important and measurable effects. Hence, the analyst should be aware of such effects and consider the properties of the situation when executing cost-benefit analysis.

The remainder of our study proceeds as follows. Section 2 summarizes the experimental design and the empirical results for the initial anchoring field experiment. Section 3 describes the experimental design and results for the bilateral market field experiment. Section 4 concludes.

\section{Experiment Design and Results: Valuation Experiment}

Our first field experiment was conducted at sportscard tradeshows in Virginia, USA, where we set up booths similar to those of professional dealers in the sportscard market. Our subjects include both ordinary consumers (non-dealers) and professional sports memorabilia dealers. Non-dealer subjects attended the show as consumers and their presence in the marketplace indicates an existing interest in sports memorabilia. The non-dealers voluntarily approached the experimenter's booth to take part in the protocol. Dealer subjects were approached at their own booths prior to the opening of the show and asked to participate in the study. Informed consent was obtained from all participants before introducing them to the protocol. In the terminology of Harrison and List (2004), we implemented a framed field experiment.

Table 1 presents the $2 \times 2$ design we employed in our first experiment; treatments varied by type of subject (dealer or non-dealer) and type of good. The first good is an unopened pack of Upper Deck NFL cards, very recently released to the market. Importantly, since it was a recent release, consumers and dealers had little idea about the equilibrium price since production figures and demand were unknown. As with any unopened pack of collectible cards, the value of the contents are uncertain, since different player's cards have different values. The pack we used had an additional element that emphasized the lottery-like aspect of the unopened deck. These card packs had a small probability (approximately 2\%) of containing a special trading card with a swatch of fabric from a player's jersey worn during an actual NFL game. The market value for such cards depends upon the player and year, but in general is not well established at the time of pack release. During our experiment, one subject was able to sell a "jersey card" for \$15-approximately three times the value of his own estimate of the original pack's value - to a card dealer. Combining the novelty of the good with this greater value 
uncertainty, we intended to give the anchoring protocol its best chance to succeed with a common market good.

Our second good is a large jar of unsalted, shelled peanuts, which was chosen as a common good for which consumers may have an established value. Only the dealers in the market might anticipate trading this good at the sportscard show, however. Thus we denote it as the "unexpected" good for ordinary consumers. iv The recent theoretical exercise of Köszegi and Rabin (2008) that examines mistakes in implementing preferences provides the underpinnings for why such expectations might play an important role in observed behavior. One interpretation of their work is that behavior will be more anomalous in situations that present themselves as surprises.

In implementing the protocol, data were collected by a monitor working one-onone with each subject. Each subject was endowed with one, and only one, of the two goods. The endowed good was rotated randomly based on the time the non-dealer approached the table, but we intentionally oversampled consumers receiving peanuts, since pilot data showed that the variances were largest in this case, and our theory suggests that we were more likely to find considerable effects valuing this good. After receiving the good, subjects were told that they would be asked two questions about selling the good back to the experimenter, after which a coin flip would determine which response was binding. It was emphasized that they would keep either the good or cash, and that their valuation responses and the random process would determine the outcome.

The anchoring protocol was initiated by asking the subjects to write the last three digits of their social security number on the provided questionnaire (see the Appendix for a copy of the questionnaire.) Subjects were then asked the first valuation question: whether they would sell the good back for the price derived from the last three digits of their social security number. For example, if the last three digits of their social security number were 123, their associated question was: would you accept $\$ 1.23$ to sell the good back to us? Of course, this offer price was clearly uninformative, having been derived from a number that was known only to the subjects.

In the second valuation task, we elicited willingness-to-accept (WTA) compensation for the endowed good using the Becker-DeGroot-Marschak (BDM) mechanism (Becker, DeGroot, and Marschak, 1964). Our BDM protocol used a bag 
containing 41 paper slips, upon each of which was a price. Prices ranged from $\$ 0.00$ $\$ 10.00$ in 25 cent increments. It was explained to the subjects that we wanted to know the minimum compensation that they required for parting with the good with which they had been endowed. Since we were not interested in testing the incentive properties of the BDM, our protocol included an explanation that the optimal strategy was to offer one's true minimum acceptable level of compensation.

After making their BDM offer, a coin was flipped to determine which choicethe dichotomous choice or the BDM response-would be executed. If the dichotomous choice question was selected, subjects who answered "no" kept the good, and those who said "yes" sold the good for the SSN value rounded up to the nearest quarter of a dollar. If the BDM mechanism was chosen, a bid price was drawn randomly from the bag. If the bid was greater than or equal to the subject's offer, they were paid the bid amount and the good was returned to the experimenter; otherwise they received no monetary payment and kept the good. In all cases, subjects were asked to fill out a short survey before the account was settled. Survey responses are used as control variables in the regressions reported below.

Our protocol is similar to classic examples in the psychology literature (see the citations provided in footnote 2), and more recent work in both economics and psychology including that of Ariely, Lowenstein, and Prelec 2003, and recent replications of the market good component of their study (Bergman et al. 2010; Fudenberg, Levine, and Manniadis, 2012). ${ }^{\mathrm{v}}$ The original explanation for the prevalence of anchoring is due to Tversky and Kahneman (1974, p. 1128) who argue that "people make estimates by starting from an initial value that is adjusted to yield a final answer", and that "adjustments are typically insufficient." More recent work links insufficient adjustment to the need for cognitive effort (Epley and Gilovich, 2006; Bergman et al., 2010; see also Oechssler et al., 2009). These findings emphasize the contribution of the field setting provides for the understanding of anchoring, since both intuition and evidence from previous studies suggest that the heterogeneity in market experience is associated with differences in cognitive costs for valuation tasks (List and LuckingReiley, 2000).

\section{Experiment Results}


Summary statistics for the anchoring experiments are presented in Table 2. Fortytwo percent of our subjects were sportscard dealers, and 65 percent of subjects were randomly endowed with the jar of peanuts. The WTA (offer) and random anchor (soc) both varied widely, between $\$ 0-\$ 10$ and $\$ 0.09$ - $\$ 9.99$, respectively. Average experience with the sportscard market (mktyrs) was 15 years; our sample consisted primarily of men.

Before we begin with the results summary, we should note that overall, 19 percent of subjects provided inconsistent responses to the two valuation queries. The inconsistencies were exhibited by subjects who stated a minimum WTA less (greater) than the DC offer that they had initially refused (accepted). For both subject groups, the majority of inconsistencies (74 percent) were found in the peanut treatment, and those exhibiting inconsistencies had less market experience than those who were consistent. ${ }^{\mathrm{vi}}$ Approximately 3 percent provided a minimum WTA exactly equal to their anchors. The remaining 78 percent provided consistent responses in which WTA was different from the anchoring value. For completeness, we present the results with and without the inconsistent responders in the sample.

Perusal of the data provides a first result:

Result 1: In the aggregate data, anchoring does not affect economic valuations.

Preliminary evidence for this result can be found in Table 3. To begin, we use a simple null hypothesis that the BDM valuation responses (offer) are independent of the random anchor (soc). Following Ariely, Loewenstein, and Prelec (2003), we split the aggregate sample by median social security number. Row 1 in Table 3 contains the pooled data summary. In this case, for the entire sample, those with high social security numbers place a sell value of $\$ 4.41$ on average, whereas those with a low social security number place a sell value of $\$ 4.17$ on the good. A similar data pattern is observed in the data set that excludes inconsistent subjects: a selling price of $\$ 4.46$ versus $\$ 4.24$. While the data tendencies are directionally in accord with the anchoring hypothesis, a Mann-Whitney test reveals that the difference is not statistically significant for either the overall sample $(p=0.32)$, or the consistent responders $(p=0.35)$, at conventional levels. 
To provide an additional test of the null hypothesis, we regress offer on the social security value and the control variables age, education, gender, and income which were gathered via the survey. Model 1 in Table 4 provides empirical estimates for all data and the subset of consistent responders. ${ }^{\text {vii }}$ Evidence of the ability to anchor market participants would arise from a positive coefficient on soc that is both economically and statistically significant. We find that the unconditional results summarized in Table 3 are supported by the regression model. Coefficients are small in magnitude and have pvalues of 0.73 and 0.82 for all and consistent responders, respectively. Thus, even after controlling for individual specific observables, we find that the offer is not unduly influenced by the uninformative anchor in the pooled data.

Clearly, however, data aggregation could be masking important heterogeneities. Upon parsing the data at a finer level, we find our next result:

Result 2A: There is some evidence that ordinary consumers are influenced by the random anchor when valuing the unexpected good.

Result 2B: There is little evidence that market professionals can be anchored for either good.

Table 3 provides the first evidence to support Result 2. First, examining the data by subject type, our non-parametric tests yield evidence that the anchor has a modest effect on values for non-dealers. Inspection at the level of treatments makes clear that the result is associated only with their valuation of the peanuts: in this case, we find a marginally significant effect of the social security number among the consistent responders at the $\mathrm{p}=$ 0.09 level. Yet, as Table 3 indicates, there is little evidence of anchoring for non-dealers valuing the sportscard pack or for dealers valuing either good.

Summarizing, we find no evidence of anchoring for dealers, but some evidence of anchoring for non-dealers. To explore the role of experience in more detail, we pool the dealer and non-dealer data and examine the effect of years of experience in the market. The conclusion from this analysis we present as Result 3.

Result 3A: For subjects who have one year or less of experience in the marketplace, we find considerable evidence of anchoring.

Result 3B: For subjects with more one year of experience in the marketplace, we find no evidence of anchoring. 
We begin discussion of result 3 by noting that sample sizes are small at low levels of market experience. However, we discover interesting tendencies in the data that are important for the broader questions raised by the anchoring literature. We find considerable evidence of anchoring in the responses of the 14 subjects who have one year or less of experience in the market. Again splitting the sample around the median SSN, we find that the Mann-Whitney test yields a statistically significant difference $(p=0.08)$ for new market participants that is consistent with the existence of anchored responses. Among this group, the mean offer for those above (below) the median SSN is 6.00 (4.49), a difference of $\$ 1.50$, or $33 \%$. Furthermore, removing an inconsistent individual who refused to sell at the SSN price of \$9.19 and then offered \$0.50 in the BDM yields an anchoring effect that is statistically significant at the $\mathrm{p}<0.02$ level. However, among subjects with more than one year of experience we find no significant effects of the anchoring protocol.

Model 2 in Table 4 provides regression results that inform results 2 and 3. The specification includes indicator variables for the dealer subject pool, the peanut treatment (nuts) and for new market participants (new - indicating subjects with one year or less experience in the sportscard markets). Interactions of treatment and experience variables with the social security anchor are also included as well as the demographic controls used in Model 1.

As in the pooled results, the coefficient on soc is not statistically significant supporting the nonparametric tests for the non-dealers when valuing the sportscards - the baseline in the regression model. The same results hold for the linear combinations of soc when interacted with treatment indicator variables. ${ }^{\text {vii }}$ The fact that the $s o c$ variable in combination with soc $\times$ nuts is not significant $(\mathrm{p}=0.51$ (all), $\mathrm{p}=0.64$ (consistent)) detracts from the robustness of Result $2 \mathrm{~A}$ regarding the anchoring of peanut valuations by non-dealers.

With regard to market experience, however, the nonparametric results are supported by parametric regression. The soc and soc $\times$ new coefficients are jointly significant for both all and consistent respondents. ${ }^{\text {ix }}$ The magnitude of the effect is economically significant. As noted in the discussion of the nonparametric results, the difference across median SSN yields a $33 \%$ percent increase in WTA for those with the 
high anchor. The relevant coefficients suggest that a one percent change in the anchor yields a reflect a $0.536(0.565)$ percent change in WTA for all (consistent) respondents.

The coefficients can be interpreted in the context of the anchoring and adjustment model of Tversky and Kahneman (1974). Their model suggests that people first consider the value of the anchor and then move, though often incompletely, toward what would be their unanchored response. Since a coefficient of zero implies complete adjustment, the measured coefficient of 0.536 , in the model with all respondents, implies that $1-.536=$ 0.464 is the magnitude of the adjustment towards the true value. ${ }^{\mathrm{x}}$ While the caveat regarding the small sample remains, the result does suggest that this is an area in which additional research is warranted. In conjunction with results from ALP (2003) who showed that arbitrary initial valuations could demonstrate coherence over time, our findings suggest that the question of whether initial innocuous cues influence even experienced consumers is one that deserves further study. Our second experiment provides an initial examination of this question in the field, in a multi-lateral bargaining setting.

\section{Experiment Design and Results: Bilateral Market Experiment}

Whether, and to what extent, the anchoring affects observed above influence the operation of markets is an open issue that undoubtedly depends critically on the market institution. For example, making use of the Walrasian tatonnement mechanism, Becker (1962) proved that several fundamental features of economic analysis, such as correctly sloped supply and demand schedules, may result even when agents are irrational, serving to sufficiently relax the utility-maximizing assumption inherent in economic modeling. Similarly, using zero-intelligence traders, Gode and Sunder (1993) illustrate that the efficiency of the double-auctions institution derives largely from its structure rather than from individual learning.

In this section, we explore how anchoring in markets affects market outcomes in multi-lateral bargaining contexts. Our market treatments are similar to Chamberlin (1948), as extended recently to naturally occurring markets by List and Millimet (2008),

of which the design description follows. In our bilateral market sessions, each participant's experience typically followed four steps: (1) consideration of the invitation 
to participate in an experiment, (2) learning the market rules, (3) actual market participation, and (4) conclusion of the experiment and exit interview.

In Step 1, before the market opened, a monitor approached dealers at the sportscard show and inquired about their interest in participating in an experiment that would take about 45 minutes. Since most dealers are accompanied by at least one other employee, it was not difficult to obtain their agreement after it was explained to them that they could earn money during the experiment. Non-dealers were recruited from people milling around the marketplace.

Once the prerequisite number of dealers (sellers) and non-dealers agreed to participate, monitors thoroughly explained the experiment's rules in Step 2. The experimental instructions were standard, and borrowed from Davis and Holt (1993, pp. 47-55) with the necessary adjustments. Before continuing, a few key aspects of the experimental design should be highlighted. First, all individuals were informed that they would receive a $\$ 10$ participation payment upon completion of the experiment. In addition, following Smith (1964), to ensure that marketers would engage in a transaction at their reservation prices, we provided a $\$ 0.05$ commission for each executed trade for both buyers and sellers.

Second, the non-dealers were informed that the experiment consisted of five periods and that they would be buyers in the experiment. In each of the five periods, we used Smith's (1976) induced value mechanism by providing each buyer with a "buyer's card" containing a number - known only to that buyer - representing the maximum price that he or she would be willing to pay for one unit of the commodity. Dealers were informed that they would be sellers in the market and, in each of the five periods, that each would be given a "seller's card" containing three sequential numbers - known only to that seller - representing the minimum price that he or she would be willing to sell up to three units. Importantly, both buyers and sellers were informed that this information was strictly private and that reservation values would change each period. They were also informed about the number of buyers and sellers in the market (explained more fully below) and informed that agents may have different values. 
Third, the monitor explained how earnings (beyond the participation and commission payments) would be determined. The difference between the contract price and the maximum reservation price determined the market earnings of buyers; the difference between the contract price and the minimum reservation price determined sellers' earnings. Several examples illustrated the irrationality associated with buying (selling) the commodity above (below) the induced value.

Fourth, the homogeneous commodities used in the experiment were 1982 Topps Ben Oglivie baseball cards, upon which decorative moustaches had been drawn, thereby rendering the cards valueless outside of the experiment. Consequently, the assignment given to buyers was clear: enter the marketplace and purchase the Oglivie "moustache" card for as little as possible. Likewise, the task confronting sellers was equally as clear, and an everyday occurrence: sell the Oglivie "moustache" card for as much as possible. The cards and participating dealers were clearly marked to ensure buyers had no trouble finding the commodity of interest. Finally, buyers and sellers engaged in two five-minute practice periods to gain experience with the market.

In Step 3, subjects participated in the bilateral market. Each market session consisted of five market periods, each lasting five minutes. After each five-minute period, a monitor privately gathered the buyers and gave each a new buyer's card; a different monitor privately gave each seller a new seller's card. Note that throughout the market process careful attention was paid to prohibit discussions between sellers (or buyers) that could induce collusive outcomes. Much like the early writers in this area, we wanted to give neoclassical theory its best chance to succeed. Step 4 concluded the experiment, where subjects were paid their earnings in private.

We follow this procedure in each of three treatments. Treatment 1 is the baseline, which we includes 12 (4) buyers (sellers). The buyers have unitary demand whereas the sellers have up to 3 items they can sell. Figure 1 presents buyer and seller induced values, which are taken from Davis and Holt (1993, pp. 14-15). In Figure 1, each step represents a distinct induced value that was given to buyers (demand curve) and sellers (supply curve). The extreme point of the intersection of the buyer and supplier rent areas 
in Figure 1 yields $\$ 37$ in rents per period, which occurs at the static price/quantity of Price $=\$ 13-\$ 14$ and Quantity $=7$.

Treatments 2 and 3, which are the novelty of this experiment, augment Treatment 1 by announcing a previous price that was realized in past experiments. This price is announced to all experimental participants in the following form: "in a previous experiment identical to this one, the first transaction occurred at a price of $\$ X$." Previous literature (e.g., Simonson and Drolet, 2004) suggests that once the decision to buy (or sell) has been taken, value judgments "are most susceptible to influence by anchors relating to market prices." Indeed, summarizing the results from four experiments, Simonson and Drolet (2004) support this reasoning and highlight the importance of the source of uncertainty as a moderator of susceptibility to anchoring effects. Thus, given that our buyers and sellers have certainly taken the step to be buyers or sellers of their good, anchoring the source of uncertainty is important.

In Treatment 2 we announce only one price realization (either a high or a low price), and this announcement takes place directly before market period 1 commences. No extra information is given beyond that given in Treatment 1 from that point onwards. When announcing a high (low) price, we use the second step on the aggregate demand (supply) function: $\$ 18(\$ 9)$, which were each observed as the first transaction price in previous experiments. Due to symmetry, this price is $\$ 4$ from the equilibrium price boundary of $\$ 14(\$ 13)$.

In Treatment 3, we announce a distinct high or low price directly before each of the five market periods commences. The high price signal is drawn randomly from integers on the uniform distribution $[15,18]$; the low price signal is also randomly drawn from integers on a uniform distribution, but over $[9,12]$. Accordingly, putting these two treatments together, we can explore both short and long run effects of price anchors. Our usage of announced random anchors as previous market outcomes is directly at the heart of the source of uncertainty in these markets. By appropriately choosing plausible realized prices (taken from our previous experiment to avoid deception), we give anchoring its best shot because this announced price might contain important information pertaining to the underlying equilibrium price (indeed, by rewarding the entire source of 
price variation to anchoring we overestimate the power of anchoring). Alternatively, by following the literature and using the same induced value schedules across all five market periods, our tests represent a demanding one for anchoring.

\section{Experiment Results}

Table 5 contains summary statistics for the experimental data. We gathered data from 3 baseline sessions, 6 'Treatment 2' sessions ( 3 high signal and 3 low signal), and 6 'Treatment 3' sessions (3 high signal and 3 low signal). Given that there are sixteen unique subjects in each session, our entire design includes data drawn from 240 subjects. Entries in Table 5 provide summary price (quantity) data in the top (bottom) panel. A first insight is that the baseline treatment yields results that suggest the predictive power of supply and demand functionals. This result is in line with previous research, and points to the power of the simple situation of supply and demand curves. ${ }^{x i}$ Perusal of the data summary for the various treatments yields a first formal comparative static finding:

\section{Result 4: Price and quantity realizations in bilateral trading markets}

\section{are influenced by anchors, but the effect is transient}

A first piece of evidence to support Result 4 is that prices realized for the first market trade are linked to the anchor. Whereas the average price in the high anchor Treatment 2 is $\$ 17.70$, the average price is only $\$ 9.50$ in the low anchor Treatment 2. These differences are statistically significant at conventional levels using a Wilcoxon signed rank test. While the average price differences for the low and high anchor Treatment 2 remain in the first few periods, by period 3 the prices have reasonably converged. Any remaining price differences are small in periods 4 and 5 of Treatment 2 . These data patterns suggest that the initial anchor does not have important long-run effects on prices.

Treatment 3 data provide a different test in that agents receive a fresh signal at the beginning of each market period. Similar to the Treatment 2 data, in this case we again find that in the early periods the signal (high or low) influences prices. For example, the initial trade is $\$ 16.30$ (\$13.30) in the high (low) treatment, and the first few periods show that prices in the high anchor treatment are above those observed in the low anchor treatment. Yet, the signals lose their power in the latter periods, where we find that little 
difference in prices exists across the high and low anchor treatments. Interestingly, in a regression model that uses the observed price as the regressand, and the signal, the market period, and the interaction of signal and market period as regressors, we find that in the early periods the signal has a significant influence, but by period 3 the signal no longer has an influence on the market transaction prices. This result suggests that even in the short run anchors do not have considerable influence for those agents who are experienced with market fundamentals.

Such transient effects are also found when examining quantities traded in the market. In this case, however, there are no observed differences across the high and low price signal treatments: in each instance the market is stifled by the anchor in the early periods. This is due to one side of the market holding out for unrealistic prices, due to the random price signal. Yet, this too wanes, as by the fourth period the expected market quantity is realized in all treatments.

\section{Conclusions}

Many of the standard results of welfare economics - such as the interpretation of market surplus measures, the Pareto Efficiency of perfectly competitive market outcomes, and the rationing and allocative functions of market prices - are predicated on the notion of durable and meaningful consumer preferences. An individual demand schedule should reflect maximum willingness to pay for units of a commodity, ceteris paribus. Likewise, producer decisions should be grounded in an understanding of technology and cost that reflects the profit motive. The assumption that primals of preference and technology are well-defined and stable has immense normative significance since the correspondence between observed market behavior and underlying theoretical foundations is at the heart of the application of microeconomic theory to welfare analysis and public policy.

This assumption has been challenged over the past three decades by those who argue that agents construct their preferences during the valuation process. Our study begins by extending the investigation of anchoring — one of the modalities through which preferences may be constructed - to a field environment. The extent to which decisions might be influenced by random signals is a topic which has received less attention in the economics and business literatures (Rothschild 1973; Sterman 1989; Schoemaker 1990), 
but of course represents one of the most robust findings in experimental psychology. ${ }^{\text {xii }} \mathrm{A}$ complementary field experiment examines the effect of anchoring in a decentralized bargaining market. We view this as an initial step in exploring anchoring in a natural trading institution with salient incentives.

We find evidence that anchoring matters in the valuation exercise. Valuations of both inexperienced market participants and ordinary consumers (nonprofessionals) were affected by the anchoring protocol, although the anchor only influences ordinary consumers for the good that they did not expect to trade in this market setting. We find that experienced consumers were not influenced by the anchor for either class of goods.

The results of our bilateral trading field experiment complement the valuation exercise. We find that price and quantity realizations are influenced by anchors, but the effect is transient. Price realizations for the first market trade are significantly influenced by the anchor, but price and quantity realizations converge to neoclassical predictions by the third round of trading. Thus, potentially informative anchors appear to have little influence on aggregate market behavior in a bilateral trading experiment in either the short run, when a new signal is offered up before each trading period, or the long run when multiple rounds of trading occur after exposure to an anchor. Overall, our results provide evidence that anchoring effects are not persistent in markets with repeated opportunities to engage in exchange within a common, static trading regime. Yet, they do highlight the import that anchoring effects potentially have on cost-benefit analysis.

Methodologically, our study highlights that using field experiments and/or "special" markets (like those for sportscards) to focus on deep questions that are hard to take on with observational field data, or in markets that are more important per se, represents a useful first attempt in the field to learn about fundamental tenets of human behavior. In this spirit, continued exploration of the extent to which individuals with different levels of market experience are influenced in the valuation of both private and public goods should provide fundamental insight into basic assumptions of microeconomic theory and the functioning of various market structures. Moreover, additional research on the influence of standard valuation protocols embedded in survey questions should yield additional insights into assessment of consumer preferences for public goods, as well as provide guidance to scholars engaged in cost-benefit analysis. 
Table 1 Anchoring - Experimental Design and Sample Size

Non-Dealers Dealers Row Totals

Sportscards

Treatment 1

Treatment 2

$$
n=34
$$

$n=32$

$n=66$

Peanuts

Treatment 3

Treatment 4

$$
n=75
$$

$$
n=46
$$$$
n=121
$$

Column Totals

$$
n=109
$$

$n=78$

$N=187$ 
Table 2 Anchoring - Descriptive Subject Pool Characteristics

\begin{tabular}{lcccccccc}
\hline & \multicolumn{9}{c}{ Sportscards } \\
Variable & Mean & St.Dev & Min & Max & Mean & St.Dev & Min & Max \\
& & & & & & & & \\
\hline Offer & 3.47 & 1.56 & 0 & 6.00 & 3.80 & 2.04 & 0.50 & 10 \\
Soc & 4.73 & 2.92 & 0.09 & 9.50 & 5.05 & 2.94 & 0.26 & 9.99 \\
Sell & 0.62 & 0.49 & 0 & 1 & 0.72 & 0.46 & 0 & 1 \\
Education & 3.85 & 1.46 & 2 & 6 & 4.09 & 1.61 & 2 & 6 \\
Age & 35.97 & 12.17 & 18 & 70 & 49.19 & 13.30 & 19 & 74 \\
Gender & 0.15 & 0.36 & 0 & 1 & 0.09 & 00.30 & 0 & 1 \\
Income & 61.30 & 33.80 & 5.00 & 125.00 & 66.98 & 41.85 & 5.00 & 125.00 \\
Mktyrs & 13.06 & 7.89 & 0 & 35 & 19.23 & 15.83 & 1 & 68 \\
\hline
\end{tabular}

\section{Peanuts}

Non-Dealers

Variable Mean St.Dev Min Max

Offer

Soc

Sell

Education

Age

Gender

Income

Mktyrs

$\begin{array}{llll}4.18 & 2.11 & 0 & 10\end{array}$

$\begin{array}{llll}4.62 & 2.63 & 0.23 & 9.47\end{array}$

$0.62 \quad 0.49$

$\begin{array}{llll}4.03 & 1.61 & 2 & 6\end{array}$

$\begin{array}{llll}41.20 & 14.13 & 19 & 70\end{array}$

$0.13 \quad 0.34$

$56.41 \quad 35.07$

$\begin{array}{lll}14.24 & 10.07 \quad 0\end{array}$

$0 \quad 1$

1

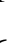

Note. The

dichor attainment ranging from incomplete grammar school to completed graduate education. Gender is defined as $1=$ female, $0=$ male. Mktyrs represents years of activity in the sportscard market. Income is in thousands of dollars. 
Table 3 Evidence of Anchoring

\begin{tabular}{|c|c|c|c|c|c|c|c|c|c|}
\hline \multirow[b]{3}{*}{ Population } & \multicolumn{5}{|c|}{ All Respondents } & \multicolumn{4}{|c|}{ Consistent Respondents } \\
\hline & Median & Mean & Mean & & $P$ & Mean & Mean & & $P$ \\
\hline & Split & $S S N$ & Offer & $N$ & Value & $S S N$ & Offer & $N$ & Value \\
\hline \multirow[t]{2}{*}{ Pooled } & High & 7.25 & 4.41 & 92 & 0.32 & 7.46 & 4.46 & 75 & 0.35 \\
\hline & Low & 2.34 & 4.17 & 91 & & 2.53 & 4.24 & 75 & \\
\hline \multirow[t]{2}{*}{ Cards } & High & 7.19 & 3.58 & 32 & 0.82 & 7.56 & 3.68 & 28 & 0.47 \\
\hline & Low & 2.40 & 3.88 & 32 & & 2.50 & 3.80 & 28 & \\
\hline \multirow[t]{2}{*}{ Nuts } & High & 7.18 & 4.99 & 60 & 0.11 & 7.40 & 5.00 & 47 & 0.13 \\
\hline & Low & 2.24 & 4.46 & 59 & & 2.54 & 4.44 & 47 & \\
\hline \multirow[t]{2}{*}{ Nondealers } & High & 6.81 & 4.33 & 53 & 0.23 & 7.06 & 4.44 & 45 & 0.09 \\
\hline & Low & 2.24 & 3.86 & 52 & & 2.35 & 3.78 & 44 & \\
\hline \multirow[t]{2}{*}{ Dealers } & High & 7.70 & 4.74 & 39 & 1.00 & 7.97 & 4.23 & 31 & 0.90 \\
\hline & Low & 2.38 & 4.78 & 39 & & 2.71 & 5.20 & 30 & \\
\hline \multirow[t]{2}{*}{ Treatment 1: Nondealers/Cards } & High & 6.96 & 3.69 & 16 & 0.96 & 7.31 & 3.78 & 14 & 0.59 \\
\hline & Low & 2.12 & 3.64 & 16 & & 2.14 & 3.54 & 14 & \\
\hline \multirow[t]{2}{*}{ Treatment 2: Dealers/Cards } & High & 7.42 & 3.62 & 16 & 0.67 & 7.81 & 3.59 & 14 & 0.77 \\
\hline & Low & 2.69 & 3.97 & 16 & & 2.85 & 4.06 & 14 & \\
\hline \multirow[t]{2}{*}{ Treatment 3: Nondealers/Nuts } & High & 6.74 & 4.54 & 37 & 0.30 & 6.94 & 4.73 & 31 & 0.09 \\
\hline & Low & 2.30 & 4.02 & 36 & & 2.44 & 3.89 & 30 & \\
\hline \multirow[t]{2}{*}{ Treatment 4: Dealers/Nuts } & High & 7.85 & 5.36 & 23 & 0.87 & 8.07 & 4.99 & 17 & 0.82 \\
\hline & Low & 2.21 & 5.50 & 23 & & 2.62 & 5.94 & 16 & \\
\hline
\end{tabular}

Note. The Mann-Whitney test was used to test the null hypothesis that the distribution of offers did not differ above and below the median SSN anchor. The test was conducted on the entire sample and subpopulations by treatments and factors, as well as a subset that were consistent in their responses to the valuation questions. Among this group we find support for the alternative hypothesis among the non-dealers and particularly those who valued peanuts. 
Table 4 Evidence of Anchoring - GLS Estimates

\section{Dependent Variable}

Offer

Soc

Soc x New

Dealer

Soc x Dealer

Nuts

Soc x Nuts

Soc x Dealer x Nuts

Education

Age

Gender

Income

Constant
Model 1 All Consistent

0.022

(0.057)

0.016
$(0.061)$

$(0.061)$
Model 2

All Consistent

$\begin{array}{ll}0.109 & 0.102 \\ (0.080) & (0.084) \\ -0.440 & 0.508 \\ (0.860) & (1.010) \\ 0.427^{* *} & 0.448^{* *} \\ (0.177) & (0.184) \\ 1.145^{*} & 1.485^{* *} \\ (0.614) & (0.729) \\ -0.331^{* *} & -0.378^{* * *} \\ (0.133) & (0.141) \\ 0.538 & 0.647\end{array}$

$0.538 \quad 0.647$

$\begin{array}{ll}(0.528) & (0.611)\end{array}$

$-0.031 \quad-0.055$

$\begin{array}{ll}(0.114) & (0.124)\end{array}$

$0.258^{* *} \quad 0.279$ **

$(0.124) \quad(0.132)$

$0.232 * * \quad 0.299 * *$

$(0.099) \quad(0.115)$

$0.035^{* * *} \quad 0.033^{* * *}$

$\begin{array}{ll}(0.011) & (0.013)\end{array}$

$0.339 \quad 0.416$

$\begin{array}{ll}(0.462) & (0.503)\end{array}$

$-0.169 * * \quad-0.237 * *$

$\begin{array}{ll}(0.081) & (0.092)\end{array}$

$1.622 * \quad 1.640 *$

$(0.901) \quad(0.975)$

\begin{tabular}{lcccc}
\hline $\mathrm{N}$ & 172 & 143 & 172 & 143 \\
$\mathrm{R}^{2}$ & 0.060 & 0.063 & 0.184 & 0.199 \\
$\mathrm{~F}$ & 2.30 & 1.95 & 2.78 & 2.58 \\
Prob $>\mathrm{F}$ & 0.090 & 0.047 & 0.002 & 0.004 \\
\hline
\end{tabular}

Note. Standard errors are in parentheses beneath the coefficients and are calculated using the Hulbert-White estimator. Statistical significance is indicated by: $* \mathrm{p}<.10, * * \mathrm{p}<.05, * * * \mathrm{p}<.01$. The dependent variable offer is the willingness-to-accept elicited from the BDM protocol. Soc is the anchor value derived from the subjects SSN. New is 1 if market experience is less than or equal to 1 year and zero otherwise. Model 1 includes control variables for individual characteristics and Model 2 adds treatment indicators and interactions. The interaction $s o c \mathrm{x}$ new provides evidence of the effectiveness of the anchoring protocol on those with the least market experience. 


\section{Baseline}

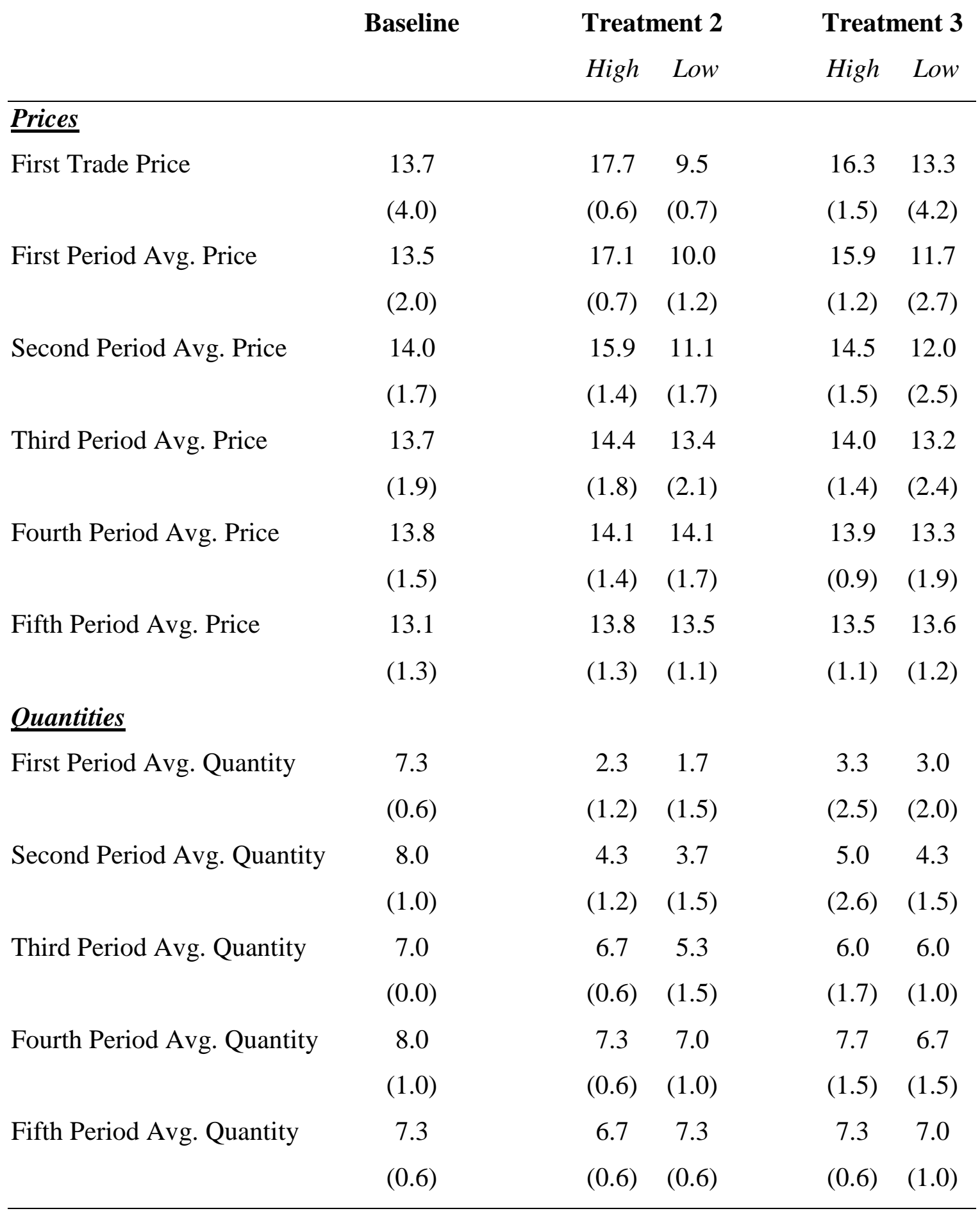

Notes: First trade price is the first executed transaction in the session. For the baseline this price is the average over the 3 sessions; for Treatments 2 and 3 this price is the average over the 5 sessions. The other figures represent the average of the session averages in each of the given periods. High and Low represent the high and low signal treatments. Standard deviations are in parentheses underneath the means. 


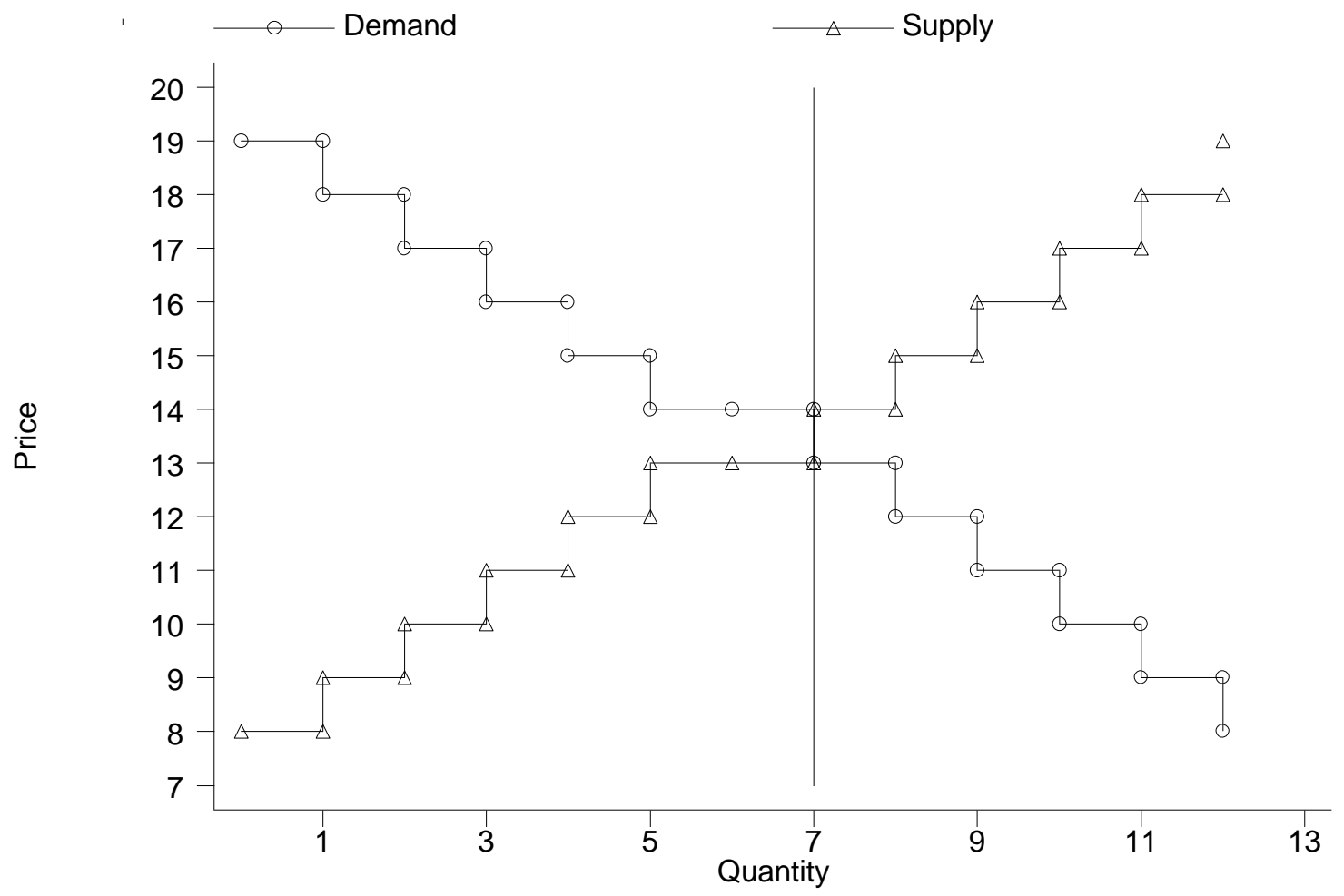

Figure 1. Buyer and seller induced values for market experiment 
Appendix

Detailed procedures: anchored valuation question

In this experiment we will ask you three questions.

First what are the last three digits of your social security number?

Please write them here

You have been given good " $\mathrm{X}$ " and we will now ask you two questions about selling it. After answering the two questions, we will flip a coin and your answer to one of the questions will be carried out. If the coin turns up heads your answer to the first question is used and you will either keep the good or sell it based on your answer. If the coin turns up tails we will use the second question, and you will either keep the good or sell it depending on your answer to that question.

\section{Question 1.}

You have the opportunity to sell " $\mathrm{X}$ " back to us for $\$ S . S N$, the value of the last three digits of your social security number converted into dollars and cents.

Would you accept $\$ S . S N$ to sell the good back to us? Yes

For question 2 you will tell us the price at which you are willing to sell the good. Details of the procedure are on the next page. 
Detailed instructions: BDM Individual Choice Elicitation Method

Welcome to Lister's Auctions. You have been given good " $\mathrm{X}$ " and have the opportunity to either keep it or sell it back for a price that will be determined in the following way.

I am holding a bag that contains 20 slips numbered 1 through 20 . You are welcome to verify this. I am going to ask you to write on the offer sheet a price at which you are willing to sell $\mathrm{X}$. If the number I draw from the bag, lets call it $\$ \mathrm{~A}$, is greater than or equal to the price you have written down you will receive $\$ \mathrm{~A}$ and return the good to me. If $\$ \mathrm{~A}$ is less than the price you have written on the offer sheet then you keep the good.

With this method of determining the selling price the best thing for you to do is use your true value for the good as the selling price. Let's see why this is true. First consider the case where you offer to sell for less than your true value. Suppose you offer $\$ B$, which is less than you really value the good. If the draw of $\$ A$ is greater than $\$ \mathrm{~B}$ but still less than your true value you must sell the good for a price that is less than your value. Your loss is the difference between $\$ A$, the price you receive, and your value, which is greater than $\$ A$.

Suppose instead that you write on your offer sheet a price greater than your true value. Let's call your offer price \$C. If my draw of \$A is greater than your true value but less than \$C, you keep the good when you would have preferred to sell it and receive $\$ A$. The amount of your loss is the difference between your value and $\$ A$. Do you have any questions about the selling process?

Please indicate the price at which you are willing to sell the good: 


\section{References}

Ariely, D., U. Gneezy, G. Loewenstein and N. Mazar. 2009. "Large Stakes and Big Mistakes," Review of Economic Studies 76(2): 451-69.

Ariely, D., G. Loewenstein, and D. Prelec. 2003. "Coherent Arbitrariness": Stable Demand Curves without Stable Preferences. Quarterly Journal of Economics 118(1): 73-105.

Bateman, Ian J., D. Burgess, W. G. Hutchinson, and D. I. Matthews. 2008. Learning Design Contingent Valuation (LDCV): NOAA Guidelines, Preference Learning, and Coherent Arbitrariness. Journal of Environmental Economics and Management 55: 127-41.

Becker, G. S. 1962. Irrational Behavior and Economic Theory. Journal of Political Economy 70: 1-13.

Becker, G. M., M. H. DeGroot, and J. Marschak. 1964. Measuring Utility by a Single Response Sequential Method. Behavioral Science 9(3): 226-32.

Bergman, Oscar, Tore Ellingsen, Magnus Johannesson, and Cicek Svensson. 2010. Anchoring and Cognitive Ability. Economics Letters 107: 66-68.

Bettman, J.R., M.F. Luce, and J.W. Payne. 1998. Constructive Consumer Choice Processes. The Journal of Consumer Research 25(3): 187-217.

Breusch, T. S. and A. R. Pagan 1979. A Simple Test for Heteroscedasticity and Random Coefficient Variation. Econometrica 47(5): 1287-94.

Chamberlin, E. H. 1948. An Experimental Imperfect Market. Journal of Political Economy 56: 95-108.

Chapman, G. B., and E. J. Johnson. 1994. The Limits of Anchoring. Journal of Behavioral Decision Making 7: 223-242.

Chapman, G. B., and E. J. Johnson. 2002. Incorporating the Irrelevant: Anchors in 
Judgments of Belief and Value. Heuristics and Biases: The Psychology of Intuitive Judgment. Eds. T. Gilovich, D. Griffin, and D. Kahneman, 120-38. Cambridge, UK: Cambridge University Press.

Choi, James J., Emily Haisley, Jennifer Kurkoski, and Cade Massey. 2012. Small Cues Change Savings Choices. NBER Working Paper 17843.

Davis, D. D. and C. A. Holt. 1993. Experimental Economics Princeton University Press: Princeton, NJ.

Einhorn, H. J., R. M. Hogarth. Ambiguity and Uncertainty in Probabilistic Inference. Psychological Review, 1985, (92), 433-461.

Englich, B. and T. Mussweiler. 2001. Sentencing under Uncertainty: Anchoring Effects in the Courtroom. Journal of Applied Social Psychology 31(7): 1535-51.

Epley, N. 2004. A Tale of Tuned Decks? Anchoring as Adjustment and Anchoring as Activation. The Blackwell Handbook of Judgment and Decision Making. Eds. D.J. Koehler \& N. Harvey, Oxford, UK: Blackwell Publishers.

Epley, N. and T. Gilovich. 2006. The Anchoring and Adjustment Heuristic: Why the Adjustments Are Insufficient. Psychological Science 17(4):311-8.

Fudenberg, Drew, David K. Levine, and Zacharias Manniadis. On the Robustness of Anchoring Effects in WTP and WTA Experiments. American Economic Journal: Microeconomics 4(2): 131-145.

Furnham, Adrian and Hua Chu Boo. 2011. A Literature Review of the Anchoring Effect. The Journal of Socio-Economics 40 (2011): 35-42.

Gneezy, U. and G. Wu "The uncertainty effect: When a risky prospect is valued less than its worst possible outcome," Quarterly Journal of Economics, 2006, 121, 4, 1283 1309. 
Gode, D. K. and S. Sunder. 1993. Allocative Efficiency of Markets with ZeroIntelligence Traders: Market as a Partial Substitute for Individual Rationality. Journal of Political Economy 101(1): 119-37.

Green, D., K. E. Jacowitz , D. Kahneman, and D. McFadden. 1998. Referendum Contingent Valuation, Anchoring, and Willingness to Pay for Public Goods. Resource and Energy Economics 20: 85-116.

Harrison, Glenn W. and John A. List. 2004. Field Experiments. Journal of Economic Literature 42: 1009-1055.

Hoeffler, S., and D. Ariely. 1999. Constructing Stable Preferences: A Look Into Dimensions of Experience and Their Impact on Preference Stability. Journal of Consumer Psychology 8, no. 2: 113-39.

Holmes, T. P. and R. A. Kramer. 1995. An Independent Sample Test of Yea-

Saying and Starting Point Bias in Dichotomous-Choice Contingent Valuation. Journal of Environmental Economics and Management 29: 121-132.

Johnson, E. J. and D. A. Schkade. 1989. Bias in Utility Assessment: Further Evidence and Explanations. Management Science 35(4):406-24.

Köszegi, B. and M. Rabin. 2008. Revealed Mistakes and Revealed Preferences. The Foundations of Positive and Normative Economics, Andrew Caplin and Andrew Schotter (editors), Oxford University Press (2008), Chapter 8, pp. 193-209.

Levitt, S., List., J., 2007. What do laboratory experiments measuring social preferences reveal about the real world? Journal of Economic Perspectives 21(2), 153-174.

List, J.A. and D. Lucking-Reiley. 2000. Demand reduction in multiunit auctions: evidence from a sportscard field experiment. American Economic Review 90(4): 961-972. 
List J.A. and D.L. Millimet. 2008. The Market: Catalyst for Rationality and Filter of Irrationality, The B.E. Journal of Economic Analysis \& Policy (Frontiers): Vol. 8: Iss. 1 (Frontiers), Article 47.

Oechssler. J., Roider, A., and Schmitz, P.W. 2009. Cognitive abilities and behavioral biases, Journal of Economic Behavior \& Organization 72(1): 147-152.

Rothschild, M. 1973. Models of Market Organization with Imperfect Information: A Survey. Journal of Political Economy 81(6): 1283-1308.

Schkade, D. A., E. G. Johnson. Cognitive Processes in Preference Reversals. Organizational Behavior and Human Decision Processes, 1989, 44 (2), 203-231.

Schoemaker, P. J. H. 1990. Strategy, Complexity and Economic Rent Management Science 36(10): 1178-92.

Simonson, I., A. Drolet. Anchoring Effects on Consumers' Willingness-to-Pay and Willingness-to-Accept. Journal of Consumer Research, 2004, 31 681-90.

Slovic, P. 1995. The Construction of Preference. American Psychologist 50(5): 364-71.

Smith, V. L. 1964. The Effect of Market Organization on Competitive Equilibrium. Quarterly Journal of Economics 78: 181-201.

Smith, V. L. 1976. Experimental Economics: Induced Value Theory. American Economic Review Paper and Proceedings 66: 274-79.

Sterman, J. D. 1989. Modeling Managerial Behavior: Misperceptions of Feedback in a Dynamic Decision Making Experiment. Management Science 35(3): 321-39.

Switzer, F. S., and J. A.Sniezek, 1991. Judgment Processes in Motivation: Anchoring and Adjustment Effects on Judgment and Behavior. Organizational Behavior and Human Decision Processes 49: 208-29.

Tversky, A., and D. Kahneman. 1974. Judgment under Uncertainty: Heuristics and Biases. Science 185: 1124-31. 
White, H. 1980. A heteroskedasticity-consistent covariance matrix estimator and a direct test for heteroskedasticity. Econometrica 48: 817-30.

Wright, W. F. and U. Anderson. 1989. Effects of Situation Familiarity and Financial Incentives on Use of the Anchoring and Adjustment Heuristic for Probability Assessment. Organizational Behavior and Human Decision Processes 44(1): 6882. 
* Author names and affiliations deleted for purposes of review.

${ }^{\mathrm{i}}$ For those interested Tolkien fans, only 1,500 copies of the first edition were printed. An Arizona buyer recently purchased a first edition copy for $\$ 65,000$ from a New York bookseller. See http://www.abebooks.com/docs/10-anniversary/powers-10.shtml.

ii There are a number of valuation exercises that show the effectiveness of an anchoring manipulation (see, e.g, Johnson and Schkade, 1989; Slovic, 1995; Bettman, Luce, and Payne, 1998; Green et al., 1998; Hoeffler and Ariely, 1999, Ariely, Loewenstein, and Prelec, 2003; Bateman et al. 2008, Bergman et al. 2010). Taken together, these results suggest strong effects, even over goods that subjects have experience in consuming. Yet, anchoring results run much deeper than valuation exercises. For instance, anchoring has been found to be important in answers to factual questions (Tversky and Kahneman, 1974; Epley and Gilovich, 2006), the estimation of probabilities (Wright and Anderson, 1989), predictions of future performance (Switzer and Sniezek, 1991), social judgments (Chapman and Johnson, 2002), legal decisions (Englich and Mussweiler, 2001), and savings rates (Choi et al. 2012), to name a few. It also represents a key component of theories of preference reversal (Schkade and Johnson, 1989) and the effect of ambiguity on probability judgments (Einhorn and Hogarth, 1985). The interested reader should see Epley (2004) for an excellent overview of the literature and the theories underlying how and why anchoring influences decisions. Furnham and Boo (2011) also provide a thorough review.

iii Not all studies report strong anchoring effects. Among the few that report weak or negligible effects, however, the same concerns about the power of incentives can arise. For example, Chapman and Johnson (1994) use extreme anchors to test the limits of anchoring in a classroom experiment in which all responses are hypothetical. In a laboratory setting, Fudenberg, Levine, and Maniadis (2012) largely fail to replicate the strong anchoring results of Ariely, Loewenstein, and Prelec's (2003) classroom study. The use of large participation fees in the laboratory study resulted in compensation that is largely unrelated to the anchoring protocol. A strength of our experimental design is that incentives parallel those occurring in a market setting. Each subject is anchored on one good and the compensation is associated entirely with responses to the anchoring protocol. Further strengths of the field design are discussed in the text.

${ }^{\text {iv }}$ We note that dealers entering the market likely expect to be offered a trade with just about anything in this market. For instance, one of the coauthors was once offered a pair of "personally worn" Marilyn Monroe panty hose in trade for a Ken Griffey Jr. rookie card. He politely declined.

${ }^{v}$ In addition to the anchoring of market goods Ariely, Loewenstein and Prelec (2003) implemented an anchoring protocol that elicited WTA for novel distasteful beverages. In this setting they observed initial anchoring and subsequent responses that were coherent given the initial anchored value. The authors characterize the responses as "coherent arbitrariness" and argue that their evidence implies that anchored responses may have a residual influence on market behavior among experienced consumers.

${ }^{v i}$ A Mann-Whitney test of differences in market experience, measured in market years, across groups of consistent and inconsistent responders yielded $p=0.08$. Further, roughly three-fourths of the inconsistent 
responders made offers to sell at a price greater than their social security number when they had previously agreed to sell at that price. The attempt to sell at a price higher than the anchor value suggests that the inconsistent subjects misunderstood the properties of the BDM mechanism, and may have believed they were in a bargaining situation, despite our instruction to report their true value.

vii In our regressions, we exclude two influential observations from both non-dealer models. These individuals refuse to sell at anchor values greater than $\$ 9.00$ but then make offers of fifty cents or less in the BDM, indicating confusion or inattention. Standard errors are calculated using the White sandwich estimator (White, 1980) since the Breusch-Pagan test detects heteroscedasticity (Breusch and Pagan, 1979).

viii Consistent with the fact that dealer valuations are somewhat higher for those with SSN below the median, there is what appears to be a negative anchoring effect associated with the soc + soc $\times$ dealer coefficient. Given the one-sided nature of our hypothesis we believe that this is an artifact and do not believe we have discovered a new phenomenon of economic significance. The positive coefficient on soc $\times$ dealer $\times$ nuts restricts this artifact to the dealers in the sportscard treatment.

${ }^{\mathrm{ix}}$ For all (consistent) respondents the joint significance of $\operatorname{soc}$ and new yields $\mathrm{p}=0.005(\mathrm{p}=0.004)$.

${ }^{\mathrm{x}}$ The insignificant coefficients for the other treatments imply complete adjustment from the anchored value.

${ }^{x i}$ We also gathered data in a treatment that used an anchor of $\$ 13.50$, the midpoint of the equilibrium prediction of the supply and demand curve intersection. These data did not significantly differ from the baseline treatment data, suggesting that this market can yield efficient outcomes with or without anchors present.

xii One should take care not to jump to inference concerning underlying preferences from these experiments too quickly. In many cases, important properties of the situation change across exercises, and changes in these properties themselves might induce agents to behave differently (see Levitt and List, 2007). 